\title{
Solving time fractional Burgers' and Fisher's equations using cubic B-spline approximation method
}

\author{
Abdul Majeed ${ }^{1 *}$, Mohsin Kamran' ${ }^{1}$, Muhammad Kashif Iqbal ${ }^{2}$ and Dumitru Baleanu $u^{3,4,5}$
}

\section{"Correspondence:}

abdulmajeed@ue.edu.pk

'Department of Mathematics,

Division of Science and Technology,

University of Education, Lahore,

Pakistan

Full list of author information is

available at the end of the article

\begin{abstract}
This article presents a numerical algorithm for solving time fractional Burgers' and Fisher's equations using cubic B-spline finite element method. The $L 1$ formula with Caputo derivative is used to discretized the time fractional derivative, whereas the Crank-Nicolson scheme based on cubic B-spline functions is used to interpolate the solution curve along the spatial grid. The numerical scheme has been implemented on three test problems. The obtained results indicate that the proposed method is a good option for solving nonlinear fractional Burgers' and Fisher's equations. The error norms $L_{2}$ and $L_{\infty}$ have been calculated to validate the efficiency and accuracy of the presented algorithm.
\end{abstract}

Keywords: Cubic B-spline collocation method; Time fractional differential equation; Caputo's fractional derivative; Stability and convergence; Finite difference formulation

\section{Introduction}

In recent years, most of the practical problems arising in different fields of science like biology, chemistry, physics, engineering, and mathematics lead to nonlinear fractional partial differential equations [1-3]. To solve these nonlinear mathematical models, several techniques have been developed. However, still it is noted that a large number of nonlinear fractional differential equations that are encountered in these areas are very difficult to solve numerically. The numerical solution of such kind of models has been the key interest of researchers due to their wide range of applications in real life.

In open literature, a lot of work is available on solving linear/nonlinear partial/fractional partial differential equations numerically by means of different techniques. The authors in [4] used the Galerkin finite element method to solve fractional diffusion and fractional diffusion wave equations. A B-spline collocation method was used by [5] for numerical treatment of fractional diffusion and fractional diffusion wave equations. Mehbobi et al. [6] employed radial basis functions to solve time fractional nonlinear Schrodinger equation. The authors in $[7,8]$ investigated the approximate solutions of fractional telegraph equation by means of radial basis functions and a fully discrete local discontinuous Galerkin method respectively. Nonlinear Fisher's equation has been studied in $[9,10]$ by means of

(c) The Author(s) 2020. This article is licensed under a Creative Commons Attribution 4.0 International License, which permits use sharing, adaptation, distribution and reproduction in any medium or format, as long as you give appropriate credit to the original author(s) and the source, provide a link to the Creative Commons licence, and indicate if changes were made. The images or other third party material in this article are included in the article's Creative Commons licence, unless indicated otherwise in a credit line to the material. If material is not included in the article's Creative Commons licence and your intended use is not permitted by statutory regulation or exceeds the permitted use, you will need to obtain permission directly from the copyright holder. To view a copy of this licence, visit http://creativecommons.org/licenses/by/4.0/. 
variational iteration method (VIM) and homotopy perturbation method (HPM) respectively. Yokus and Kaya [11] employed the Cole-Hopf transformation and the expansion methods to solve time fractional Burgers' equation. Jin et al. [12] presented the numerical analysis of nonlinear sub-diffusion equations. Li et al. [13] proposed Galerkin's finite element approach for numerical solution of nonlinear time fractional parabolic equations. A Galerkin scheme based on Crank-Nicolson method has been used in [14] for solving nonlocal coupled parabolic problems. A cubic B-spline based Galerkin approach has been applied by Iqbal et al. [15] to solve Schrodinger equation. In general, the finite element method based on third degree basis spline functions has not been widely used for solving time fractional problems and this will be the focus of our paper. In this work, we consider the following generalized time fraction partial differential equation:

$$
\begin{aligned}
& \frac{\partial^{\alpha} y(z, t)}{\partial t^{\alpha}}+\eta(y(z, t))^{q}(y(z, t))_{z}-v(y(z, t))_{z z}-\beta y(z, t)[1-y(z, t)]=H(z, t), \\
& 0 \leq z \leq L, 0 \leq t \leq T, 0<\alpha \leq 1,
\end{aligned}
$$

subject to the term of initial and boundary conditions

$$
\begin{aligned}
& y(z, 0)=g(z), \quad 0 \leq z \leq L, \\
& y(0, t)=g_{1}(t), \quad y(L, t)=g_{2}(t), \quad 0 \leq t \leq T,
\end{aligned}
$$

where $\eta, v, \beta$ are parameters, $H(z, t)$ is the source term, $q$ is a positive integer, and $g(z)$, $g_{i}(t)$ are known functions. There are several interpretations of fractional order derivatives; however, we use Caputo's definition as follows [16]:

$$
\frac{\partial^{\alpha} y(z, t)}{\partial t^{\alpha}}= \begin{cases}\int_{0}^{t} \frac{\partial^{\alpha} y(z, s)}{\partial s^{\alpha}} \frac{(t-s)^{M-\alpha-1}}{\Gamma(M-\alpha)} d s, & M-1<\alpha<M, \\ \frac{\partial^{\alpha} y(z, t)}{\partial t^{\alpha}}, & M=\alpha,\end{cases}
$$

where $M$ is the smallest integer exceeding $\alpha$.

If we take $\eta=0$ and $q=1$, equation (1) takes the following form:

$$
\frac{\partial^{\alpha} y(z, t)}{\partial t^{\alpha}}-v(y(z, t))_{z z}-\beta y(z, t)[1-y(z, t)]=H(z, t) .
$$

The above equation, known as time fractional Fisher's equation [17], is a mathematical model for the temporal spatial propagation of a virile gene in an infinite domain. This model is also used in chemical kinetics [18], auto catalytic chemical reactions [19], neurophysiology [20], nuclear reactor theory [21], branching Brownian motion process [22], and flame propagation [23].

If we set $\beta=0$ and $q=1$ in (1), it reduces to Burgers' equation

$$
\frac{\partial^{\alpha} y(z, t)}{\partial t^{\alpha}}+\eta y(z, t)(y(z, t))_{z}-v(y(z, t))_{z z}=H(z, t)
$$

This is the simplest nonlinear model in fluid dynamics for diffusive waves. It appears in various physical issues such as viscous sound waves, waves in fluid-filled viscous elastic pipes, magneto hydrodynamic waves in a medium with finite electrical conductivity [24] and one-dimensional turbulence. 


\section{Description of numerical method}

Let us partition the temporal domain $[0, T]$ into $N$ subintervals $\left[t_{n}, t_{n+1}\right]: t_{n}=n \Delta t, n=$ $0,1,2, \ldots, N$, where $\Delta t=\frac{T}{N}$. The fractional time derivative defined in equation (4) for $M=$ 1 can be approximated at $t=t_{n+1}$ by using L1 formula as follows [25]:

$$
\frac{\partial^{\alpha} y\left(z, t_{n+1}\right)}{\partial t^{\alpha}}=\frac{(\Delta t)^{-\alpha}}{\Gamma(2-\alpha)} \sum_{\xi=0}^{n} \sigma_{\xi}\left[y\left(z, t_{n-\xi+1}\right)-y\left(z, t_{n-\xi}\right)\right]+E_{\Delta t}^{n+1},
$$

where $\sigma_{\xi}=(\xi+1)^{1-\alpha}-\xi^{1-\alpha}$ and $E_{\Delta t}^{n+1}$ denotes the truncation error at $(n+1)$ th time stage. This error is bounded s.t.

$$
\left|E_{\Delta t}^{n+1}\right| \leq \varsigma(\Delta t)^{2-\alpha}, \quad \varsigma \in \mathbb{R}^{+}
$$

Moreover, $\sigma_{\xi}$ s have the following attributes [25]:

- $\sigma_{0}=1$ and $0<\sigma_{\xi}, \xi=1,2, \ldots, n$;

- $\sigma_{0}>\sigma_{1}>\sigma_{2}>\cdots>\sigma_{\xi}, \sigma_{\xi} \rightarrow 0$ as $\xi \rightarrow \infty$;

- $\sum_{\xi=0}^{n}\left(\sigma_{\xi}-\sigma_{\xi+1}\right)+\sigma_{n+1}=\left(1-\sigma_{1}\right)+\sum_{\xi=1}^{n-1}\left(\sigma_{\xi}-\sigma_{\xi+1}\right)+\sigma_{n}=1$.

Using (7) together with theta weighted scheme, equation (1), with $q=1$, takes the following shape:

$$
\begin{gathered}
\gamma \sum_{\xi=0}^{n} \sigma_{\xi}\left[y^{n-\xi+1}-y^{n-\xi}\right]+\theta\left[\eta\left(y y_{z}\right)^{n+1}-v\left(y_{z z}\right)^{n+1}-\beta y^{n+1}\right] \\
+(1-\theta)\left[\eta\left(y y_{z}\right)^{n}-v\left(y_{z z}\right)^{n}-\beta y^{n}\right]=H^{n+1}+\beta F^{n}
\end{gathered}
$$

where $\gamma=\frac{(\Delta t)^{-\alpha}}{\Gamma(2-\alpha)}, y^{n}=y\left(z, t_{n}\right)$, and $F^{n}=\left(y\left(z, t_{n}\right)\right)^{2}$. Now, we use the following formula to linearize the term $\left(y y_{z}\right)^{n+1}[26]$ :

$$
\left(y y_{z}\right)^{n+1}=y^{n+1} y_{z}^{n}+y^{n} y_{z}^{n+1}-y^{n} y_{z}^{n}
$$

Using (9) in (8) for $\theta=1 / 2$, we get

$$
\begin{aligned}
\phi^{n} y^{n+1}+\varphi^{n}\left(y_{z}\right)^{n+1}-v\left(y_{z z}\right)^{n+1}= & (2 \gamma+\beta) y^{n}+\nu\left(y_{z z}\right)^{n} \\
& -2 \gamma \sum_{\xi=1}^{n} \sigma_{\xi}\left[y^{n-\xi+1}-y^{n-\xi}\right]+\digamma^{n+1},
\end{aligned}
$$

where $\phi^{n}=2 \gamma-\beta+\eta\left(y_{z}\right)^{n}, \varphi^{n}=\eta y^{n}$, and $\digamma^{n+1}=H^{n+1}+\beta F^{n}$.

Now, we partition the spatial domain $[0, L]$ into $R$ subintervals $\left[z_{r}, z_{r+1}\right]: z_{r}=r h, r=$ $0,1,2, \ldots, R$, where $h=\frac{L}{R}$.

Let $Y(z, t)$ be the cubic B-spline solution to the semi-discretized scheme $(10)$ at $(n+1)$ th time level s.t.

$$
Y\left(z, t_{n+1}\right)=\sum_{j=-1}^{R+1} D_{j}\left(t_{n+1}\right) B_{j}(z)
$$


where $D_{j}\left(t_{n+1}\right)$ s are unknowns to be identified and $B_{j}(z)$ are third degree basis spline functions defined as follows [27]:

$$
B_{j}(z)=\frac{1}{6 h^{3}} \begin{cases}\left(z-z_{j}\right)^{3}, & \text { if } z \in\left[z_{j}, z_{j+1}\right), \\ h^{3}+3 h^{2}\left(z-z_{j+1}\right)+3 h\left(z-z_{j+1}\right)^{2}-3\left(z-z_{j+1}\right)^{3}, & \text { if } z \in\left[z_{j+1}, z_{j+2}\right), \\ h^{3}+3 h^{2}\left(z_{j+3}-z\right)+3 h\left(z_{j+3}-z\right)^{2}-3\left(z_{j+3}-z\right)^{3}, & \text { if } z \in\left[z_{j+2}, z_{j+3}\right), \\ \left(z_{j+4}-z\right)^{3}, & \text { if } z \in\left[z_{j+3}, z_{j+4}\right), \\ 0, & \text { otherwise. }\end{cases}
$$

In this way, equation (10) can be written as follows:

$$
\begin{aligned}
\phi^{n} Y^{n+1}+\varphi^{n}\left(Y_{z}\right)^{n+1}-v\left(Y_{z z}\right)^{n+1}= & (2 \gamma+\beta) Y^{n}+v\left(Y_{z z}\right)^{n} \\
& -2 \gamma \sum_{\xi=1}^{n} \sigma_{\xi}\left[Y^{n-\xi+1}-Y^{n-\xi}\right]+\digamma^{n+1} .
\end{aligned}
$$

For the sake of simplicity, we denote $Y\left(z_{r}, t_{n+1}\right)$ by $Y_{r}^{n+1}$ and discretize equation (13) along the spatial grid as follows:

$$
\begin{aligned}
\phi_{r}^{n} & Y_{r}^{n+1}+\varphi_{r}^{n}\left(Y_{z}\right)_{r}^{n+1}-v\left(Y_{z z}\right)_{r}^{n+1} \\
\quad & (2 \gamma+\beta) Y_{r}^{n}+v\left(Y_{z z}\right)_{r}^{n}-2 \gamma \sum_{\xi=1}^{n} \sigma_{\xi}\left[Y_{r}^{n-\xi+1}-Y_{r}^{n-\xi}\right]+\digamma_{r}^{n+1}, \quad r=0,1,2, \ldots, R .
\end{aligned}
$$

From (11) and (12), we obtain

$$
\left\{\begin{array}{l}
Y_{r}^{n+1}=D_{r-1}^{n+1}\left(\frac{1}{6}\right)+D_{r}^{n+1}\left(\frac{4}{6}\right)+D_{r+1}^{n+1}\left(\frac{1}{6}\right), \\
\left(Y_{z}\right)_{r}^{n+1}=D_{r-1}^{n+1}\left(\frac{-1}{2 h}\right)+D_{r}^{n+1}(0)+D_{r+1}^{n+1}\left(\frac{1}{2 h}\right), \\
\left(Y_{z z}\right)_{r}^{n+1}=D_{r-1}^{n+1}\left(\frac{1}{h^{2}}\right)-D_{r}^{n+1}\left(\frac{2}{h^{2}}\right)+D_{r+1}^{n+1}\left(\frac{1}{h^{2}}\right) .
\end{array}\right.
$$

By plugging (15) into (14), we obtain the following set of equations:

$$
a_{r}^{n} D_{r-1}^{n+1}+b_{r}^{n} D_{r}^{n+1}+c_{r}^{n} D_{r+1}^{n+1}=\omega_{r}^{n}, \quad r=0,1,2, \ldots, R, n=0,1,2, \ldots, N,
$$

where $a_{r}^{n}=\frac{\phi_{r}^{n}}{6}-\frac{\varphi_{r}^{n}}{2 h}-\frac{v}{h^{2}}, b_{r}^{n}=\frac{4 \phi_{r}^{n}}{6}+\frac{2 v}{h^{2}}, c_{r}^{n}=\frac{\phi_{r}^{n}}{6}+\frac{\varphi_{r}^{n}}{2 h}-\frac{v}{h^{2}}$, and $\omega_{r}^{n}=(2 \gamma+\beta) Y_{r}^{n}+v\left(Y_{z z}\right)_{r}^{n}-$ $2 \gamma \sum_{\xi=1}^{n} \sigma_{\xi}\left[Y_{r}^{n-\xi+1}-Y_{r}^{n-\xi}\right]+\digamma_{r}^{n+1}$.

Two more relations are obtained from end conditions (3):

$$
\left\{\begin{array}{l}
D_{-1}^{n+1}\left(\frac{1}{6}\right)+D_{0}^{n+1}\left(\frac{4}{6}\right)+D_{1}^{n+1}\left(\frac{1}{6}\right)=\left(g_{1}\right)^{n+1}, \\
D_{R-1}^{n+1}\left(\frac{1}{6}\right)+D_{R}^{n+1}\left(\frac{4}{6}\right)+D_{R+1}^{n+1}\left(\frac{1}{6}\right)=\left(g_{2}\right)^{n+1} .
\end{array}\right.
$$


Equations (16)-(17) can be expressed in a matrix form as follows:

$$
\left[\begin{array}{cccccccc}
\frac{1}{6} & \frac{4}{6} & \frac{1}{6} & 0 & \cdots & 0 & 0 & 0 \\
a_{0}^{n} & b_{0}^{n} & c_{0}^{n} & 0 & \cdots & 0 & 0 & 0 \\
0 & a_{1}^{n} & b_{1}^{n} & c_{1}^{n} & \cdots & 0 & 0 & 0 \\
\vdots & \vdots & \vdots & \vdots & \ddots & \vdots & \vdots & \vdots \\
0 & 0 & 0 & 0 & \cdots & a_{R}^{n} & b_{R}^{n} & c_{R}^{n} \\
0 & 0 & 0 & 0 & \cdots & \frac{1}{6} & \frac{4}{6} & \frac{1}{6}
\end{array}\right]\left[\begin{array}{c}
D_{-1}^{n+1} \\
D_{0}^{n+1} \\
D_{1}^{n+1} \\
\vdots \\
D_{R}^{n+1} \\
D_{R+1}^{n+1}
\end{array}\right]=\left[\begin{array}{c}
\left(g_{1}\right)^{n+1} \\
\omega_{0}^{n+1} \\
\omega_{1}^{n+1} \\
\vdots \\
\omega_{R}^{n+1} \\
\left(g_{2}\right)^{n+1}
\end{array}\right] .
$$

The above system is solved for $\left(D_{-1}^{n+1}, D_{0}^{n+1}, D_{2}^{n+1}, \ldots, D_{R+1}^{n+1}\right)^{T}$. Using this vector in (11), we get the approximate solution at $(n+1)$ th time level for $n=0,1,2, \ldots, N$. However, to start the iterative process, we need to evaluate the initial vector. The initial conditions of the problem under consideration will help to compute the initial vector $\left(D_{-1}^{0}, D_{0}^{0}, D_{2}^{0}, \ldots, D_{R+1}^{0}\right)^{T}$ as follows: Discretization of (2) results in the following set of equations [28]:

$$
\left\{\begin{array}{l}
Y_{z}\left(z_{0}, t_{0}\right)=g_{z}\left(z_{0}\right) \\
Y\left(z_{r}, t_{0}\right)=g_{z}\left(z_{r}\right) \text { for } r=0,1,2, \ldots, R \\
Y_{z}\left(z_{R}, t_{0}\right)=g_{z}\left(z_{R}\right)
\end{array}\right.
$$

Using (15) in the above system, we get the following matrix form:

$$
\left[\begin{array}{cccccccc}
\frac{-1}{2 h} & 0 & \frac{1}{2 h} & 0 & \cdots & 0 & 0 & 0 \\
\frac{1}{6} & \frac{4}{6} & \frac{1}{6} & 0 & \cdots & 0 & 0 & 0 \\
\vdots & \vdots & \vdots & \vdots & \ddots & \vdots & \vdots & \vdots \\
0 & 0 & 0 & 0 & \cdots & \frac{1}{6} & \frac{4}{6} & \frac{1}{6} \\
0 & 0 & 0 & 0 & \cdots & \frac{-1}{2 h} & 0 & \frac{1}{2 h}
\end{array}\right]\left[\begin{array}{c}
D_{-1}^{0} \\
D_{0}^{0} \\
\vdots \\
D_{R}^{0} \\
D_{R+1}^{0}
\end{array}\right]=\left[\begin{array}{c}
\left(g_{z}\right)_{0}^{0} \\
g_{0}^{0} \\
\vdots \\
g_{R}^{0} \\
\left(g_{z}\right)_{R}^{0}
\end{array}\right] .
$$

This above equation, $(N+3) \times(N+3)$ matrix system, is solved using Thomas algorithm. For numerical computations, MATLAB software has been used.

\section{Stability analysis}

For the sake of simplicity, we discuss the stability of the presented scheme for the following linear force free case of problem (1):

$$
\frac{\partial^{\alpha} y(z, t)}{\partial t^{\alpha}}+\eta(y(z, t))_{z}-v(y(z, t))_{z z}-\beta y(z, t)=0 .
$$

Employing the method described in Sect. 3 for $\theta=1$, the fully discretized scheme for equation (21) is given by

$$
\begin{aligned}
\kappa_{1} D_{r-1}^{n+1}+\kappa_{2} D_{r}^{n+1}+\kappa_{3} D_{r+1}^{n+1} & \\
= & \frac{\gamma}{6} D_{r-1}^{n}+\frac{4 \gamma}{6} D_{r}^{n}+\frac{\gamma}{6} D_{r+1}^{n} \\
& \quad-\gamma \sum_{\xi=1}^{n} \sigma_{\xi}\left[\frac{1}{6}\left(D_{r-1}^{n-\xi+1}-D_{r-1}^{n-\xi}\right)+\frac{4}{6}\left(D_{r}^{n-\xi+1}-D_{r}^{n-\xi}\right)+\frac{1}{6}\left(D_{r+1}^{n-\xi+1}-D_{r+1}^{n-\xi}\right)\right],
\end{aligned}
$$


where

$$
\kappa_{1}=\frac{\gamma}{6}-\frac{\beta}{6}-\frac{\eta}{2 h}-\frac{v}{h^{2}}, \quad \kappa_{2}=\frac{4 \gamma}{6}-\frac{4 \beta}{6}+\frac{2 v}{h^{2}}, \quad \kappa_{3}=\frac{\gamma}{6}-\frac{\beta}{6}+\frac{\eta}{2 h}-\frac{v}{h^{2}} .
$$

Now, we bring the Fourier method into play for stability analysis. Let $\Omega^{n}$ denote the growth factor and $\tilde{\Omega}^{n}$ be its estimated value. The error $\Upsilon^{n}$ at $n$th time stage is given by

$$
\Upsilon^{n}=\Omega^{n}-\tilde{\Omega}^{n}
$$

where $\Upsilon^{n}=\left[\Upsilon_{1}^{n}, \Upsilon_{2}^{n}, \cdot, \Upsilon_{N-1}^{n}\right]^{T}$.

From (22), we have

$$
\begin{aligned}
\kappa_{1} \Upsilon_{r-1}^{n+1}+\kappa_{2} \Upsilon_{r}^{n+1}+\kappa_{3} \Upsilon_{r+1}^{n+1} \\
=\frac{\gamma}{6} \Upsilon_{r-1}^{n}+\frac{4 \gamma}{6} \Upsilon_{r}^{n}+\frac{\gamma}{6} \Upsilon_{r+1}^{n} \\
\quad-\gamma \sum_{\xi=1}^{n} \sigma_{\xi}\left[\frac{1}{6}\left(\Upsilon_{r-1}^{n-\xi+1}-\Upsilon_{r-1}^{n-\xi}\right)+\frac{4}{6}\left(\Upsilon_{r}^{n-\xi+1}-\Upsilon_{r}^{n-\xi}\right)+\frac{1}{6}\left(\Upsilon_{r+1}^{n-\xi+1}-\Upsilon_{r+1}^{n-\xi}\right)\right]
\end{aligned}
$$

The end conditions (2)-(3) are also satisfied by error term as follows:

$$
\Upsilon_{r}^{0}=g\left(z_{r}\right), \quad \Upsilon_{0}^{n}=g_{1}\left(t_{n}\right), \quad \Upsilon_{R}^{n}=g_{2}\left(t_{n}\right), \quad n=0,1, \ldots, N, r=1,2, \ldots, R .
$$

Now, we introduce a mesh function

$$
\Upsilon^{n}= \begin{cases}\Upsilon_{r}^{n}, & z_{r}-\frac{h}{2}<z \leq z_{r}+\frac{h}{2}, r=1: 1: R-1 \\ 0, & a \leq z \leq a+\frac{h}{2} \text { or } b-\frac{h}{2} \leq z \leq b\end{cases}
$$

Also, $\Upsilon^{n}(z)$ can be written in terms of Fourier series as follows:

$$
\Upsilon^{n}(z)=\sum_{-\infty}^{\infty} \rho_{n}(m) e^{\frac{2 m \pi \pi z}{b-a}}, \quad n=0,1, \ldots, N
$$

where

$$
\rho_{n}(m)=\frac{1}{b-a} \int_{a}^{b} \Upsilon^{n}(z) e^{\frac{-2 m \pi-a z}{b-a}} d z .
$$

$\Rightarrow$

$$
\begin{aligned}
\left\|\Upsilon^{n}\right\|_{2} & =\sqrt{\sum_{r=1}^{R-1} h\left|\Upsilon_{r}^{n}\right|^{2}} \\
& =\sqrt{\int_{a}^{a+\frac{h}{2}}\left|\Upsilon^{n}\right|^{2} d z+\sum_{r=1}^{R-1} \int_{z_{r}-\frac{h}{2}}^{z_{r}+\frac{h}{2}}\left|\Upsilon^{n}\right|^{2} d z+\int_{b-\frac{h}{2}}^{b}\left|\Upsilon^{n}\right|^{2} d z} \\
& =\int_{a}^{b}\left|\Upsilon^{n}\right|^{2} d z=\sum_{-\infty}^{\infty}\left|\rho_{n}(m)\right|^{2} \quad \text { (using Parseval's equality). }
\end{aligned}
$$


Now, we plug $\Upsilon_{r}^{n}=\rho_{n} e^{\text {erh }}$ into (24) and simplify to get the following expression:

$$
\begin{aligned}
\kappa_{1} \rho_{n+1} \mathrm{e}^{-\iota \varrho h}+\kappa_{2} \rho_{n+1}+\kappa_{3} \rho_{n+1} \mathrm{e}^{\iota \varrho h} & \\
= & \frac{\gamma}{6} \rho_{n} \mathrm{e}^{-\iota \varrho h}+\frac{4 \gamma}{6} \rho_{n}+\frac{\gamma}{6} \rho_{n} \mathrm{e}^{\iota \varrho h} \\
& \quad-\gamma \sum_{\xi=1}^{n} \sigma_{\xi}\left[\frac{1}{6}\left(\rho_{n-\xi+1}-\rho_{n-\xi}\right) \mathrm{e}^{-\iota \varrho h}+\frac{4}{6}\left(\rho_{n-\xi+1}-\rho_{n-\xi}\right)+\frac{1}{6}\left(\rho_{n-\xi+1}-\rho_{n-\xi}\right) \mathrm{e}^{\iota \varrho h}\right],
\end{aligned}
$$

where $\iota=\sqrt{-1}$ and $\varrho=\frac{2 m \pi}{b-a}$. After some simplification, (30) takes the form

$$
\rho_{n+1}=\frac{1}{\chi} \rho_{n}-\frac{1}{\chi} \sum_{\xi=1}^{n} \sigma_{\xi}\left(\rho_{n-\xi+1}-\rho_{n-\xi}\right),
$$

where $\chi=1+\frac{12 v \sin ^{2}(\varrho h / 2)-\beta h^{2}(2+\cos (\varrho h))}{\gamma h^{2}(2+\cos (\varrho h))}$. It can be noted that $|\chi| \geq 1$. Now, we make use of mathematical induction to show that $\left|\rho_{n}\right| \leq\left|\rho_{0}\right|$ for all $n$.

For $n=0,(31)$ gives

$$
\left|\rho_{1}\right|=\frac{1}{|\chi|}\left|\rho_{0}\right| \leq\left|\rho_{0}\right| \because|\chi| \geq 1
$$

Now, let $\left|\rho_{n}\right| \leq\left|\rho_{0}\right|$ for $n>0$.

Using (31), we have

$$
\begin{aligned}
\left|\rho_{n+1}\right| & \leq \frac{1}{|\chi|}\left|\rho_{n}\right|+\frac{1}{|\chi|} \sum_{\xi=1}^{n} \sigma_{\xi}\left(\left|\rho_{n-k+1}\right|-\left|\rho_{n-k}\right|\right) \\
& \leq \frac{1}{|\chi|}\left|\rho_{0}\right|+\frac{1}{|\chi|} \sum_{\xi=1}^{n} \sigma_{\xi}\left(\left|\rho_{0}\right|-\left|\rho_{0}\right|\right) \\
& \leq\left|\rho_{0}\right| \because|\chi| \geq 1 .
\end{aligned}
$$

Hence

$$
\left|\rho_{n}\right| \leq\left|\rho_{0}\right| \text { for all } n \text {. }
$$

Using (29) and (33), we have

$$
\left\|\Upsilon^{n}\right\| \leq\left|\Upsilon^{0}\right|_{2}
$$

which concludes that the proposed scheme is unconditionally stable.

\section{Convergence analysis}

In this section, we derive the uniform convergence of the proposed scheme. 
Lemma 4.1 Consider $y(z, t)$ to be the exact solution of problem (1) in $[0, L]$. Let $\tilde{Y}(z, t)$ be the unique spline approximation to (1), then $\forall t \geq 0, \exists \mu_{j}$, not depending on $h$, s.t.

$$
\left\|D^{l}(y-\tilde{Y})\right\|_{\infty} \leq \beth_{l} h^{4-l}, \quad l=0,1,2
$$

Lemma 4.2 The spline basis $B_{i}, i=-1,0, \ldots, R+1$, satisfies the inequality [29]

$$
\sum_{s=-1}^{R+1}\left|B_{s}(z)\right| \leq \frac{5}{3}, \quad 0 \leq z \leq 1 .
$$

Let $y, Y$ be the exact and approximate solutions for the given problem and $\tilde{Y}=\sum_{l=-1}^{R+1} \epsilon_{l} B_{l}$ be the spline approximation for $Y$ with the interpolation conditions $\operatorname{Ly}\left(z_{r}, t\right)=\operatorname{LY}\left(z_{r}, t\right)=$ $g\left(z_{r}, t\right), r=0: 1: R$, then

$$
L \tilde{Y}(z, t)=\tilde{g}\left(\left(z_{r}, t\right)\right.
$$

At $t=t_{n}$, the problem can be expressed in terms of difference equation $L\left(\tilde{Y}\left(z_{r}, t\right)-Y\left(z_{r}, t\right)\right)$ as follows:

$$
\begin{aligned}
\kappa_{1} \wp_{r-1}^{n+1}+\kappa_{2} \wp_{r}^{n+1}+\kappa_{3} \wp_{r+1}^{n+1} \\
=\frac{\gamma}{6} \wp_{r-1}^{n}+\frac{4 \gamma}{6} \wp_{r}^{n}+\frac{\gamma}{6} \wp_{r+1}^{n} \\
\quad-\gamma \sum_{\xi=1}^{n} \sigma_{\xi}\left[\frac{1}{6}\left(\wp_{r-1}^{n-\xi+1}-\wp_{r-1}^{n-\xi}\right)+\frac{4}{6}\left(\wp_{r}^{n-\xi+1}-\wp_{r}^{n-\xi}\right)+\frac{1}{6}\left(\wp_{r+1}^{n-\xi+1}-\wp_{r+1}^{n-\xi}\right)\right]+\digamma_{r}^{n+1},
\end{aligned}
$$

where $\wp_{r}^{n}=D_{r}^{n}-\epsilon_{r}^{n}, r=0,1, \ldots, R$.

From the end conditions, we can write

$$
\frac{1}{6} \wp_{r-1}^{n+1}+\frac{4}{6} \wp_{r}^{n+1}+\frac{1}{6} \wp_{r+1}^{n+1}=0, \quad r=0, R
$$

If $\mho_{r}^{n}=h^{2}\left[\digamma_{r}^{n}-\widetilde{\digamma}_{r}^{n}\right]$ for $r=0,1, \ldots, R$, then using Lemma (4.1), we can write

$$
\left|\mho_{r}^{n}\right|=h^{2}\left|\digamma_{r}^{n}-\widetilde{\digamma}_{r}^{n}\right| \leq \beth h^{4}
$$

Let $\mho^{n}=\max \left\{\left|\mho_{r}^{n}\right| ; 0 \leq r \leq R\right\}, \tilde{E}_{r}^{n}=\left|\wp_{j}^{n}\right|$, and $\tilde{E}^{n}=\max \left\{\left|E_{r}^{n}\right| ; 0 \leq r \leq R\right\}$.

At $n=0$, equation (36) takes the shape

$$
\kappa_{1} \wp_{r-1}^{1}+\kappa_{2} \wp_{r}^{1}+\kappa_{3} \wp_{r+1}^{1}=\frac{\gamma}{6} \wp_{r-1}^{0}+\frac{4 \gamma}{6} \wp_{r}^{0}+\frac{\gamma}{6} \wp_{r+1}^{0}+\frac{1}{h^{2}} \digamma_{r}^{1} .
$$

Making use of the initial condition, we have $E^{0}=0 \Rightarrow$

$$
\kappa_{1} \wp_{r-1}^{1}=\frac{1}{h^{2}} \digamma_{r}^{1}-\kappa_{2} \wp_{r}^{1}-\kappa_{3} \wp_{r+1}^{1} .
$$


Using small grid spacing with end conditions, we can write

$$
\widetilde{E}^{1} \leq \beth_{1} h^{2}
$$

where $I_{1}$ does not contain $h$. Using mathematical induction, we can easily prove that

$$
\widetilde{E}_{j}^{n+1} \leq I h^{2}
$$

Hence

$$
\tilde{Y}(z, t)-Y(z, t)=\sum_{r=-1}^{R+1}\left(\epsilon_{r}(t)-D_{r}(t)\right) B_{r}(z) .
$$

Using Lemma (4.2), we get

$$
\|\tilde{Y}-Y\|_{\infty} \leq \frac{5}{3} \beth h^{2} .
$$

Now

$$
\begin{aligned}
\|y-Y\|_{\infty} & \leq\|y-\tilde{Y}(z, t)\|_{\infty}+\|\tilde{Y}(z, t)-Y(z, t)\|_{\infty}, \\
\|u-U\|_{\infty} & \leq \beth_{0} h^{4}+\frac{5}{3} I h^{2}=ד h^{2},
\end{aligned}
$$

where $\rceil=\beth_{0} h^{2}+\frac{5}{3} \beth$.

Hence $\left.\|y-Y\|_{\infty} \leq\right\urcorner h^{2}+\varsigma(\Delta t)^{2-\alpha}$, where $\beth$ and $\varsigma$ are constants.

\section{Applications and discussion}

In this section, we present some numerical experiments. $L_{2}$ and $L_{\infty}$ norms are used to analyze the precision of the suggested technique.

$$
L_{2}=\left\|y_{\text {exact }}-Y\right\|_{2} \simeq \sqrt{h \sum_{r=0}^{R}\left|\left(y_{\text {exact }}\right)_{r}-Y_{r}\right|^{2}},
$$

and

$$
L_{\infty}=\left\|y_{\text {exact }}-Y\right\|_{\infty} \simeq \max _{r=0}^{R}\left|\left(y_{\text {exact }}\right)_{r}-Y_{r}\right| .
$$

Example 1 Consider the following time fractional Burgers' equation:

$$
\begin{aligned}
& \frac{\partial^{\alpha} y(z, t)}{\partial t^{\alpha}}+y y_{z}(z, t)-v y_{z z}(z, t)=H(z, t), \\
& \text { IC: } \quad y(z, 0)=0, \quad 0 \leq z \leq 1, \\
& \text { BCs: } \quad y(0, t)=0, \quad y(1, t)=-t^{\frac{3}{2}}, \quad t \geq 0,
\end{aligned}
$$

where the source term on the right-hand side is given by $H(z, t)=\frac{t^{\frac{3}{2}-\alpha}}{\Gamma\left(\frac{5}{2}-\alpha\right)} \Gamma\left(\frac{5}{2}\right) \sin \frac{3 \pi}{2}(z)+$ $\frac{3 \pi}{2} t^{3} \sin \frac{3 \pi}{2}(z) \cos \frac{3 \pi}{2}(z)+\frac{9 \pi^{2}}{4} t^{\frac{3}{2}} \sin \frac{3 \pi}{2}(z)$. 


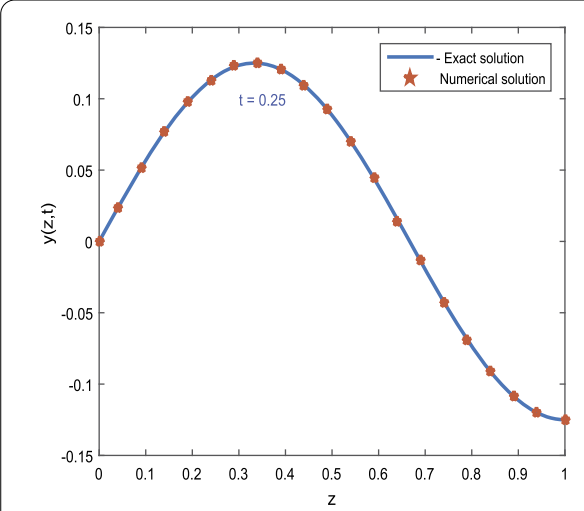

(a) For $t=0.25$

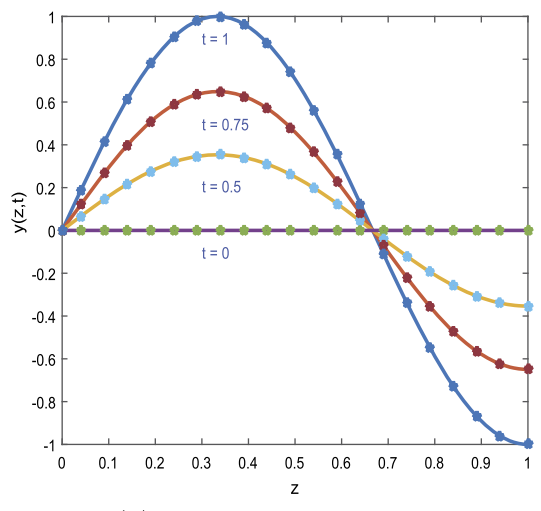

(b) For $t=0.5,0.75$ and 1

Figure 1 Numerical solution of Example 1 for $\alpha=0.5, h=0.01, \Delta t=0.00035$, and $v=1$

Figure 2 Three-dimensional view of numerical results of Example 1 for $t \in[0,1], \alpha=0.5$, step size $h=0.01$, $\Delta t=0.00035$, and $v=1$

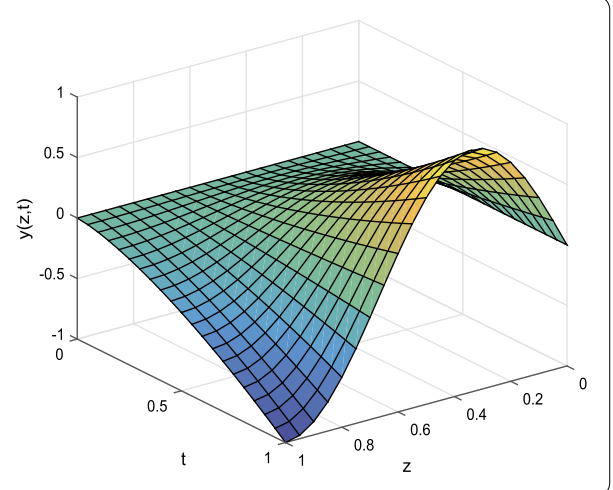

$y(z, t)=t^{\frac{3}{2}} \sin \frac{3 \pi}{2}(z)$ is the exact solution for the above initial and boundary conditions. Figure 1(a) shows the physical behavior of time fractional Burgers' equation (41) when $t=0.25$, spatial step length $h=0.01$, viscosity parameter $v=1, \Delta t=0.00035$, and $\alpha=0.5$. Figure 1(b) displays the two-dimensional plots of numerical outcomes and exact values in a single frame for distinct time stages $t=0.5,0.75$, and 1 , which clearly demonstrates that the numerical findings extracted from this system are consistent with the exact solutions. The three-dimensional plot of solution surface is shown in Fig. 2. Table 1 presents the comparison of approximate and exact solutions. We can see that the numerical results are reasonably precise. The error standards for $L_{2}$ and $L_{\infty}$ were measured and displayed in Table 2. The numerical order of convergence has been reported in Table 3.

Example 2 Consider the following time fractional Burgers' equation:

$$
\begin{aligned}
& \frac{\partial^{\alpha} y(z, t)}{\partial t^{\alpha}}+y y_{z}(z, t)-v y_{z z}(z, t)=H(z, t), \\
& \text { IC: } \quad y(z, 0)=2 z^{2}, \quad 0<z<1, \\
& \text { BCs: } \quad y(0, t)=0, \quad y(1, t)=2(t+1), \quad t \geq 0 .
\end{aligned}
$$


Table 1 Comparison of accurate and numerical solutions of Example 1 at various time levels

\begin{tabular}{|c|c|c|c|c|c|}
\hline \multicolumn{2}{|l|}{$t=0.5$} & \multicolumn{2}{|l|}{$t=0.75$} & \multicolumn{2}{|l|}{$t=1$} \\
\hline Exact & Numerical & Exact & Numerical & Exact & Numerical \\
\hline 0 & 0 & 0 & 0 & 0 & 0 \\
\hline 0.14551 & 0.14552 & 0.26734 & 0.26732 & 0.41152 & 0.41153 \\
\hline 0.27592 & 0.27593 & 0.50695 & 0.50683 & 0.78043 & 0.78042 \\
\hline 0.34624 & 0.34632 & 0.63604 & 0.63602 & 0.97923 & 0.97924 \\
\hline 0.34102 & 0.34117 & 0.62653 & 0.62655 & 0.96464 & 0.96468 \\
\hline 0.26152 & 0.26153 & 0.48041 & 0.48032 & 0.73964 & 0.73969 \\
\hline 0.12503 & 0.12504 & 0.22963 & 0.22964 & 0.34358 & 0.34358 \\
\hline-0.03885 & -0.03864 & -0.07135 & -0.07124 & -0.10978 & -0.10974 \\
\hline-0.19412 & -0.19423 & -0.35664 & -0.35666 & -0.54904 & -0.54903 \\
\hline-0.30711 & -0.30732 & -0.56423 & -0.56431 & -0.86862 & -0.86873 \\
\hline-0.35364 & -0.35364 & -0.64953 & -0.64953 & -1 & -1 \\
\hline
\end{tabular}

Table 2 Error norms for Example 1

\begin{tabular}{lll}
\hline$t$ & $L_{2}$ norm & $L_{\infty}$ norm \\
\hline 0.5 & $3.6 \times 10^{-5}$ & $2.1 \times 10^{-4}$ \\
0.75 & $2.0663 \times 10^{-5}$ & $1.2 \times 10^{-4}$ \\
1 & $1.3491 \times 10^{-5}$ & $1.1 \times 10^{-4}$ \\
\hline
\end{tabular}

Table 3 Absolute errors $\left(L_{\infty}\right)$ and order of convergence (O.C.) at $t=0.5$ for Example 1

\begin{tabular}{llllll}
\hline & $R=20$ & $R=40$ & $R=80$ & $R=160$ & $R=320$ \\
\hline$L_{\infty}$ & 0.00892 & 0.00214 & 0.000563 & 0.000120 & 0.0000258 \\
O.C. & - & 2.05943 & 1.9264 & 2.2301 & 2.21759 \\
\hline
\end{tabular}

The source term is given by

$$
H(z, t)=\frac{2 z^{2} t^{1-\alpha}}{\Gamma(2-\alpha)}+8 z^{3}(1+t)^{2}-4 v(z+1)
$$

The closed form solution to this problem is $y(z, t)=2 z^{2}(i+t)$. The exact and approximate solutions of equation (42) are shown graphically in Figs. 3. For $t=0.25, \alpha=0.5$, step size $h=0.01$, viscosity parameter $v=1$, and $\Delta t=0.00035$, the numerical solution and exact solutions are shown in Fig. 3(a); from there, we can see that both solutions agree with each other well. Figure 3(b) depicts the symmetry in both solutions for different time levels $t=$ $0.5,0.75$, and 1 . Figure 4 displays three-dimensional representation of physical behavior of the solution curve. The exact and numerical outcomes are compared in Table 4. One can see a very close agreement of the exact and approximate solutions in Table 5 . From the tabular information, it can be concluded that error reduces as the value of t varies. Table 6 shows the calculations of numerical order of convergence.

Example 3 Consider the following Fisher's equation:

$$
\begin{aligned}
& \frac{\partial^{\alpha} y(z, t)}{\partial t^{\alpha}}-v y_{z z}(z, t)-y(z, t)(1-y(z, t))=H(z, t), \\
& \text { IC: } \quad y(z, 0)=0, \quad 0<z<1, \\
& \text { BCs: } \quad y(0, t)=t^{1+\alpha}, \quad y(1, t)=0, \quad t \geq 0 .
\end{aligned}
$$




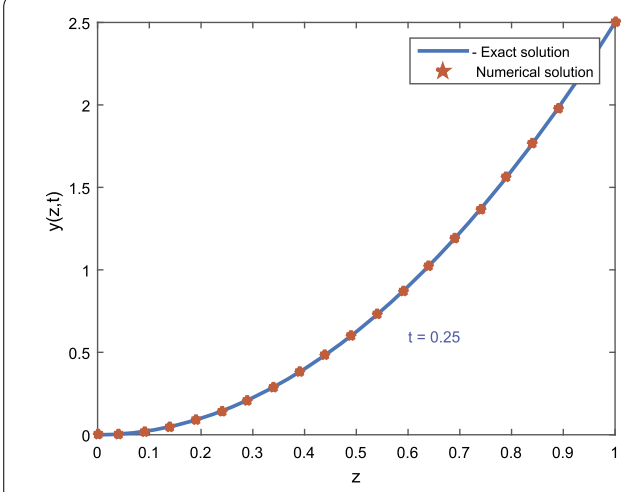

(a) For $t=0.25$

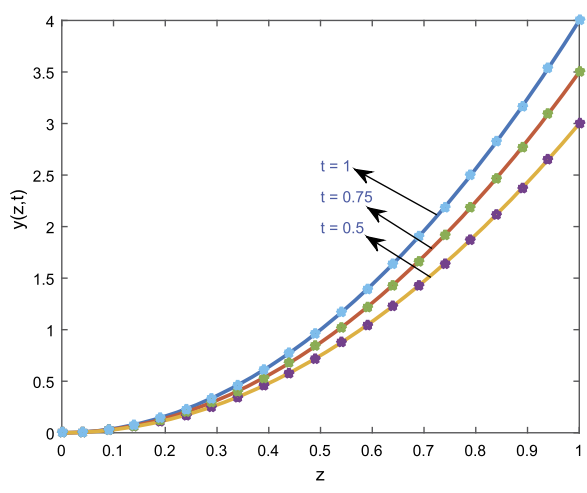

(b) For $t=0.5,0.75$ and 1

Figure 3 Numerical solution of Example 2 for $t=0.25, \alpha=0.5$, step size $h=0.01, \Delta t=0.00035$, and $v=1$

Figure 4 3D image of numerical results of Example 2 for $t \in[0,1], \alpha=0.5$, step size $h=0.01, \Delta t=0.00035$, and $v=1$

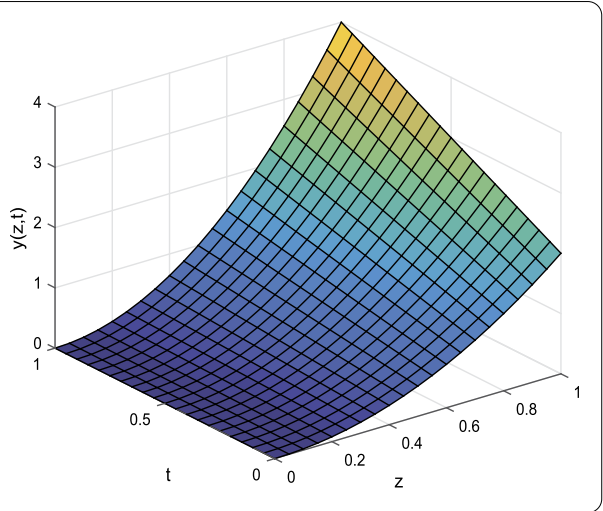

Table 4 Comparison of exact and numerical solutions of Example 2 at various times

\begin{tabular}{|c|c|c|c|c|c|}
\hline \multicolumn{2}{|l|}{$t=0.5$} & \multicolumn{2}{|l|}{$t=0.75$} & \multicolumn{2}{|l|}{$t=1$} \\
\hline Exact & Numerical & Exact & Numerical & Exact & Numerical \\
\hline 0 & 0 & 0 & 0 & 0 & 0 \\
\hline 0.02438 & 0.02435 & 0.02845 & 0.02846 & 0.03245 & 0.03247 \\
\hline 0.1083 & 0.1084 & 0.12642 & 0.12645 & 0.14447 & 0.14443 \\
\hline 0.25239 & 0.25236 & 0.29445 & 0.29443 & 0.33644 & 0.33641 \\
\hline 0.45638 & 0.45636 & 0.53247 & 0.53249 & 0.60839 & 0.60835 \\
\hline 0.72037 & 1.72035 & 0.84032 & 0.84054 & 0.96045 & 0.96039 \\
\hline 1.04435 & 0.04439 & 1.21834 & 0.21863 & 1.39243 & 1.39241 \\
\hline 1.4283 & 0.42841 & 1.66324 & 1.66322 & 1.90443 & 1.90442 \\
\hline 1.87238 & 0.87242 & 2.18445 & 2.18436 & 2.49642 & 2.49635 \\
\hline 2.37634 & 2.37643 & 2.77241 & 2.77243 & 3.16845 & 3.16873 \\
\hline 3 & 3 & 3.5 & 3.5 & 4 & 4 \\
\hline
\end{tabular}

The source term is given by

$$
\begin{aligned}
H(z, t)= & t \cos (3 \pi z)\left(1-z^{2}\right) \Gamma(2+\alpha) \\
& -t^{1+\alpha}\left[12 \pi z \sin (3 \pi z)-2 \cos (3 \pi z)-9 \pi^{2}\left(1-z^{2}\right) \cos (3 \pi z)\right] \\
& +6\left[t^{1+\alpha} \cos (3 \pi z)\left(1-z^{2}\right)\right]\left[1-\left\{t^{1+\alpha} \cos (3 \pi z)\left(1-z^{2}\right)\right\}\right] .
\end{aligned}
$$


Table 5 Error norms for Example 2

\begin{tabular}{lll}
\hline$t$ & $L_{2}$ norm & $L_{\infty}$ norm \\
\hline 0.5 & 0.1999895 & $1 \times 10^{-4}$ \\
0.75 & 0.099970998 & $2.2 \times 10^{-4}$ \\
1 & 0.0000030315 & $2.8 \times 10^{-4}$ \\
\hline
\end{tabular}

Table 6 Absolute errors $\left(L_{\infty}\right)$ and order of convergence (O.C.) at $t=0.5$ for Example 2

\begin{tabular}{llllll}
\hline & $R=20$ & $R=40$ & $R=80$ & $R=160$ & $R=320$ \\
\hline$L_{\infty}$ & 0.00984 & 0.00245 & 0.000452 & 0.000120 & 0.0000237 \\
O.C. & - & 2.00588 & 2.43839 & 1.91329 & 2.34008 \\
\hline
\end{tabular}

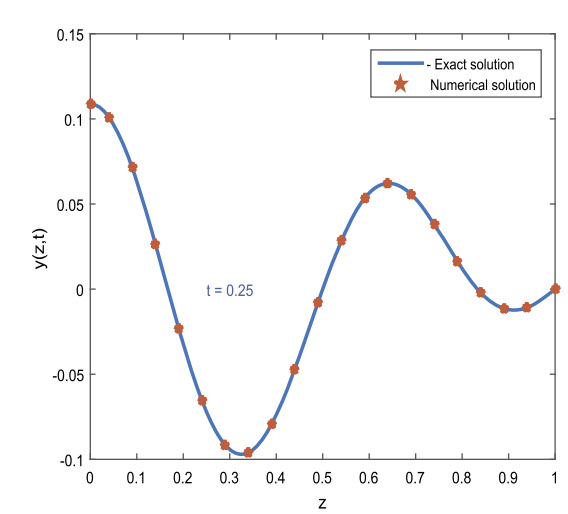

(a) For $t=0.25$

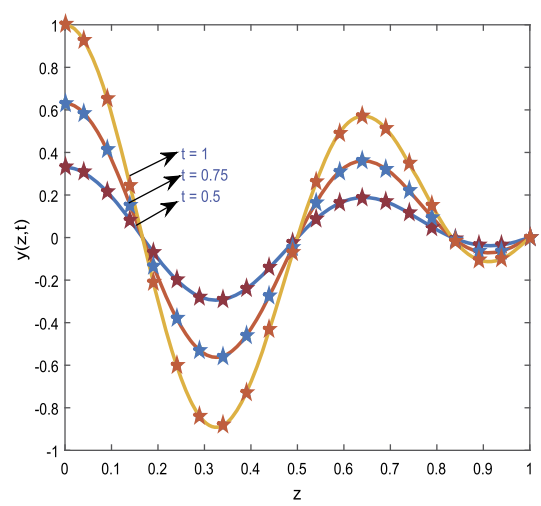

(b) For $t=0.5,0.75$ and 1

Figure 5 Numerical solution of Example 3 for variation in time at $\alpha=0.6, h=0.01, \Delta t=0.00032$, and $v=1$

Figure $63 \mathrm{D}$ view of numerical solution of Example 3 for $t \in[0,1], \alpha=0.6$, step size $h=0.01, \Delta t=0.00032$, and $v=1$

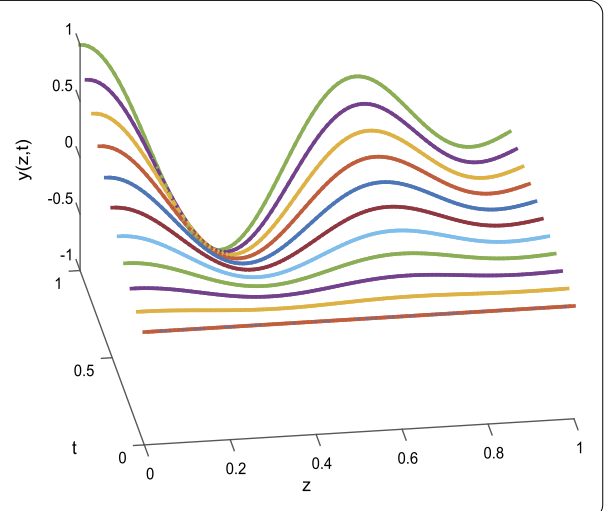

Exact solution to this problem is $y(z, t)=t^{1+\alpha} \cos (3 \pi z)\left(1-z^{2}\right)$. Figure 5 (a) shows the two-dimensional plots between numerical and approximate solutions for $\alpha=0.6, t=0.25$, $v=1, \Delta t=0.00031$, and spatial step size $h=0.01$. It is clearly seen that exact and numerical results are compatible with each other. Figure 5(b) demonstrates the symmetrical behavior of exact and approximate solutions at different time stages $t=0.5,0.75$, and 1 for fixed values of other parameters. The three-dimensional plot of solution surface is shown in Fig. 6. The comparison of exact and approximate results is given in Table 7, which shows 
Table 7 Comparison of exact and numerical solutions of Example 3 at different time level with $\alpha=0.5$

\begin{tabular}{|c|c|c|c|c|c|}
\hline \multicolumn{2}{|l|}{$t=0.5$} & \multicolumn{2}{|l|}{$t=0.75$} & \multicolumn{2}{|l|}{$t=1$} \\
\hline Exact & Numerical & Exact & Numerical & Exact & Numerical \\
\hline 0.3298769 & 0.3298321 & 0.6310997 & 0.6310887 & 1 & 1 \\
\hline 0.2163845 & 0.2163832 & 0.4139732 & 0.4139654 & 0.6559552 & 0.6559562 \\
\hline-0.0693626 & -0.0693543 & -0.1327002 & -0.1327121 & -0.2102682 & -0.2102563 \\
\hline-0.2772851 & -0.2772762 & -0.5304844 & -0.5304832 & -0.8405714 & -0.8405675 \\
\hline-0.240751 & -0.2406433 & -0.4605912 & -0.4605943 & -0.7298231 & -0.7298234 \\
\hline-0.0235904 & -0.0235912 & -0.0451317 & -0.0451321 & -0.0715129 & -0.0715123 \\
\hline 0.1613089 & 0.1613124 & 0.3086061 & 0.3086051 & 0.4889974 & 0.4889876 \\
\hline 0.1686604 & 0.1686542 & 0.3226704 & 0.3226654 & 0.5112827 & 0.5112832 \\
\hline 0.0492466 & 0.0492342 & 0.0942155 & 0.0942211 & 0.1492878 & 0.1492768 \\
\hline-0.0349107 & -0.0349112 & -0.0667891 & -0.0667355 & -0.1058297 & -0.1058324 \\
\hline 0 & 0 & 0 & 0 & 0 & 0 \\
\hline
\end{tabular}

Table 8 Absolute errors $\left(L_{\infty}\right)$ and order of convergence (O.C.) at $t=0.5$ for Example 3

\begin{tabular}{llllll}
\hline & $R=20$ & $R=40$ & $R=80$ & $R=160$ & $R=320$ \\
\hline$L_{\infty}$ & 0.0872 & 0.02137 & 0.005479 & 0.000781 & 0.000143 \\
O.C. & - & 2.02874 & 1.9636 & 2.81052 & 2.44931 \\
\hline
\end{tabular}

that both solutions are indiscriminately similar to each other. The experimental order of convergence in spatial direction has been given in Table 8.

\section{Concluding remarks}

In this work, the authors have presented a B-spline collocation method based on third degree basis spline functions and finite difference formulation for numerical investigation of time fractional Burgers' and Fisher's equations. Caputo's interpretation for time fractional derivative has been considered. The fractional time derivative has been discretized by $L 1$ formula, and B-spline functions are used to interpolate the solution along the spatial grid. The stability and convergence analysis of the proposed method is carried out. The numerical results of three different test problems reflect the applicability of the proposed scheme. The numerical outcomes validate the accuracy and efficiency of the presented method.

\section{Acknowledgements}

The authors are also indebted to the anonymous reviewers for their helpful, valuable comments and suggestions in the improvement of this manuscript.

\section{Funding}

No funding is available for this research.

Availability of data and materials

Not applicable.

\section{Competing interests}

The authors declare that they have no competing interests.

\section{Authors' contributions}

All authors equally contributed to this work. All authors read and approved the final manuscript.

\section{Author details}

'Department of Mathematics, Division of Science and Technology, University of Education, Lahore, Pakistan.

${ }^{2}$ Department of Mathematics, Government College University, Faisalabad, Pakistan. ${ }^{3}$ Department of Mathematics, Faculty of Arts and Sciences, Cankaya University, Ankara, Turkey. ${ }^{4}$ Department of Medical Research, China Medical University

Hospital, China Medical University, Taichung, Taiwan. ${ }^{5}$ Institute of Space Sciences, Magurele-Bucharest, Romania. 


\section{Publisher's Note}

Springer Nature remains neutral with regard to jurisdictional claims in published maps and institutional affiliations.

Received: 20 November 2019 Accepted: 6 April 2020 Published online: 23 April 2020

\section{References}

1. Murray, J.D.: Mathematical Biology. Springer, New York (2003)

2. Kuramoto, Y:: Chemical Oscillations Waves and Turbulence. Dover, Mineola (2003)

3. Wilhelmsson, H., Lazzaro, E.: Reaction-diffusion problems in the physics of hot plasmas (2001)

4. Esen, A., Ucar, Y., Yagmurlu, N., Tasbozan, O.: A Galerkin finite element method to solve fractional diffusion and fractional diffusion-wave equations. Math. Model. Anal. 18, 260-273 (2013)

5. Esen, A., Tasbozan, O., Ucar, Y., Yagmurlu, N.M.: A B-spline collocation method for solving fractional diffusion and fractional diffusion-wave equations. Tbil. Math. J. 8, 181-193 (2015)

6. Mohebbi, A., Mostafa, A., Dehghan, M.: The use of a meshless technique based on collocation and radial basis functions for solving the time fractional nonlinear Schrodinger equation arising in quantum mechanics. Eng. Anal. Bound. Elem. 37, 475-485 (2013)

7. Hosseini, V.R., Chen, W., Avazzade, Z.: Numerical solution of fractional telegraph equation by using radial basis functions. Eng. Anal. Bound. Elem. 38, 31-39 (2014)

8. Wei, L., Dai, H., Zhang, D., Si, Z.: Fully discrete local discontinuous Galerkin method for solving the fractional telegraph equation. Calcolo 51, 175-192 (2014)

9. Mohyud-Din, S.T., Noor, M.S.: Modified variational iteration method for solving Fisher's equations. J. Appl. Math. Comput. 31, 295-308 (2009)

10. Agirseven, D., Ozis, T:: An analytic study for Fisher type equations by using homotopy perturbation method. Comput. Math. Appl. 60, 602-609 (2010)

11. Yokus, A., Kaya, D.: Numerical and exact solutions for time fractional Burgers' equation. J. Nonlinear Sci. Appl. 10(7), 3419-3428 (2017)

12. Jin, B., Li, B., Zhou, Z.: Numerical analysis of nonlinear subdiffusion equations. SIAM J. Numer. Anal. 56, 1-23 (2018)

13. Li, D., Wu, C., Zhang, Z.: Linearized Galerkin FEMs for nonlinear time fractional parabolic problems with non-smooth solutions in time direction. J. Sci. Comput. 80, 403-419 (2019)

14. Chaudhary, S.: Crank-Nicolson-Galerkin finite element scheme for nonlocal coupled parabolic problem using the Newton's method. Math. Methods Appl. Sci. 41, 724-749 (2018)

15. Iqbal, A., Abd Hamid, N.N., Ismail, A.I.Md.: Soliton solution of Schrödinger equation using cubic B-spline Galerkin method. Fluids 4(2), Article ID 108 (2019)

16. Khalid, N., Abbas, M., Iqbal, M.K.: Non-polynomial quintic spline for solving fourth-order fractional boundary value problems involving product terms. Appl. Math. Comput. 349, 393-407 (2019)

17. Fisher, R.A.: The wave of advance of advantageous genes. Ann. Eugen. 7, 335-369 (1937)

18. Malfict, W.: Solitary wave solutions of nonlinear wave equations. Am. J. Phys. 60, 650-654 (1992)

19. Aronson, D.J., Weinberg, H.F.: Nonlinear Diffusion in Population Genetics Combustion and Never Pulse Propagation. Springer, New York (1988)

20. Tuckwell, H.C.: Introduction to Theoretical Neurobiology. Cambridge University Press, Cambridge (1955)

21. Canosa, J.: Diffusion in nonlinear multiplication media. J. Math. Phys. 10, 1862-1869 (1969)

22. Bramson, M.D.: Maximal displacement of branching Brownian motion. Commun. Pure Appl. Math. 31, 531-581 (1978)

23. Frank, D.A.: Diffusion and Heat Exchange in Chemical Kinetics. Princeton University Press, Princeton (1955)

24. Debnath, L.: Partial Differential Equations for Scientists and Engineers. Birkhäuser, Boston (1997)

25. Amin, M., Abbas, M., Iqbal, M.K., Baleanu, D.: Non-polynomial quintic spline for numerical solution of fourth-order time fractional partial differential equations. Adv. Differ. Equ. 2019(1), Article ID 183 (2019)

26. Rubin, S.G., Graves, R.A.: A cubic spline approximation for problems in fluid mechanics. Nasa TR R-436, Washington (1975)

27. Iqbal, M.K., Abbas, M., Wasim, I.: New cubic B-spline approximation for solving third order Emden-Flower type equations. Appl. Math. Comput. 331, 319-333 (2018)

28. Dag, I., Irk, D., Saka, B.: A numerical solution of Burgers equation using cubic B-splines. Appl. Math. Comput. 163 Article ID 199211 (2005)

29. Khalid, N., Abbas, M., Iqbal, M.K., Baleanu, D.: A numerical algorithm based on modified extended B-spline functions for solving time-fractional diffusion wave equation involving reaction and damping terms. Adv. Differ. Equ. 2019(1), $378(2019)$ 